La

Révolution

française

\section{La Révolution française}

Cahiers de l'Institut d'histoire de la Révolution française

$10 \mid 2016$

L'historien vivant (1789-1830)

\title{
L'expérience de la défaite, la rencontre avec l'histoire : Barnave et ses écrits historiques
}

\section{Francesco Dendena}

\section{(2) OpenEdition}

\section{Journals}

Édition électronique

URL : http://journals.openedition.org/lrf/1558

DOI : $10.4000 /$ Irf. 7558

ISSN : 2105-2557

Éditeur

IHMC - Institut d'histoire moderne et contemporaine (UMR 8066)

Édition imprimée

Date de publication : 2 mai 2016

\section{Référence électronique}

Francesco Dendena, «L'expérience de la défaite, la rencontre avec l'histoire : Barnave et ses écrits historiques », La Révolution française [En ligne], 10 | 2016, mis en ligne le 13 juin 2016, consulté le 19 avril 2019. URL : http://journals.openedition.org/Irf/1558 ; DOI : 10.4000//rf.1558

Ce document a été généré automatiquement le 19 avril 2019

(c) La Révolution française 


\title{
L'expérience de la défaite, la rencontre avec l'histoire : Barnave et ses écrits historiques
}

\author{
Francesco Dendena
}

Je tiens à remercier les lecteurs anonymes de la LRF pour leurs conseils et leurs corrections. Cet article est dédié A.V.

1 Si l'historiographie « immédiate » de la Révolution n'a gagné ses lettres de noblesse qu'au cours des toutes dernières décennies ${ }^{1}$, les écrits sur l'histoire et la Révolution d'Antoine Barnave les ont depuis très longtemps atteintes. Depuis leur première publication en 1843 ? Pas tout à fait. A l'époque, l'initiative de leur éditeur, Bérenger de la Drôme, s'inscrit dans une démarche de glorification de l'auteur plutôt qu'elle ne s'explique par la volonté de comprendre les contenus de l'œuvre. À cela concourent à la fois les liaisons personnelles de Bérenger avec Barnave ${ }^{2}$, ses convictions politiques et surtout un moment historiographique qui voit dans le héros, le primum movens du processus révolutionnaire. Michelet viendra peu après certes, mais il n'est pas encore là̀

2 Les pages introductives de Bérenger, d'ailleurs, le témoignent. Présentées comme une " une notice historique », elles sont en réalité un hommage rendu à l'homme, façonnées comme elles le sont par le souvenir de «l'enfant gâté de la Révolution ${ }^{4}$.» Courage politique, droiture morale et beauté physique : chaque aspect est mobilisé pour dresser l'image de Barnave comme d'un martyr de la « bonne » révolution de 1789 en poursuivant une opération mémorielle commencée précocement et depuis constamment entretenue, car si, dès 1799, une statue à l'entrée du Sénat avait été érigée en l'honneur du député grenoblois, par la suite ses anciens amis n'avaient pas manqué de lui rendre des hommages appuyés dans leurs mémoires ${ }^{5}$.

3 L'espace qui lui est consacré par la seconde édition de la Biographie universelle ancienne et moderne, publiée elle-aussi en 1843, est une preuve de sa renommée posthume. À la fin d'une très longue notice, le collaborateur des frères Michaud présente Barnave comme «l'un des plus sincères amis de la liberté, un homme qui fut du petit nombre des purs 
parmi les révolutionnaires ${ }^{6} . »$ En ce sens alors, les quatre volumes de Bérenger se contentent d'achever un portait que d'autres ont commencé, en confortant avec du matériel nouveau un vieil échafaudage ${ }^{7}$. C'est le protagoniste de 1789 et la victime de 1793 qu'on cherche dans ces écrits pour pouvoir le réintégrer dans l'univers politique libéral de la monarchie de Juillet ; l'historien, quant à lui, est à peine remarqué.

Il faudra attendre le début du $\mathrm{XX}^{\mathrm{e}}$ siècle et l'Histoire socialiste de la Révolution pour que cette vision ne soit radicalement dépassée, jusqu'à ce que Jean Jaurès ne trouve, dans l' Introduction à la Révolution française ${ }^{8}$, le « premier croquis du matérialisme économique de Marx ${ }^{9}$. " Les pages de Jaurès sont plus qu'un tournant interprétatif : elles constituent une véritable consécration de Barnave qui, désormais, est élevé au rang de porte-parole conscient de l'élite bourgeoise arrivée au pouvoir en 1789. Au député du Tiers État du Dauphiné revient maintenant le mérite d'avoir saisi la signification profonde de l'événement auquel il a participé, d'avoir peint la Révolution en tant que moment initiateur d'une Histoire que le marxisme se donne pour tâche d'accomplir et de dépasser. En ce sens, Jaurès écrit moins un commentaire du texte de Barnave, qu'il ne noue plutôt un dialogue avec lui en partageant la même conception du progrès historique et, dans l'analyse d'une révolution jugée inachevée, le leader socialiste peut trouver la justification de celle qui viendra.

5 Si Albert Soboul ou George Rudé se sont montrés proches de cette interprétation, François Furet ou Emmanuel Chill de leur côté l'ont seulement en partie modifiée ${ }^{10}$. Certes, leur attention s'est déplacée en amont du texte plutôt qu'en aval, en cherchant les sources d'inspiration de l'ouvrage plutôt que de s'intéresser à sa postérité comme l'avaient fait leurs collègues marxistes ${ }^{11}$. Pourtant, en dépit des différences interprétatives, se dégage une approche consensuelle qui fait de Barnave un observateur dépassionné du moment révolutionnaire, capable de développer une analyse critique des bouleversements qui ont intéressé la France, en les inscrivant dans un temps long. C'est justement ici d'ailleurs que résiderait l'intérêt et le caractère exceptionnel de l' Introduction à la Révolution barnavienne: pour être immédiat, il s'agit d'un acte historiographique pleinement abouti. À Furet d'affirmer d'abord que Barnave « aurait mis par écrit les lois qui ont gouverné à leur insu les hommes de la Révolution française, comme s'il n'y avait joué aucune rôle ${ }^{12}$ " pour ensuite ajouter :

Le caractère le plus surprenant de cette Introduction à la Révolution française, de la part d'un acteur si engagé dans l'événement dont il traite, est l'éloignement, la hauteur dont celui-ci témoigne par rapport à l'histoire qu'il vient de faire. Caractère encore plus surprenante si l'on songe aux circonstances dans lesquelles ces pages sont rédigées, dans un temps où leur auteur est désavoué par le tour pris par la Révolution, dépassé, menacé, déjà en prison. [...] Barnave contemple ce qui a été avec un détachement patricien ${ }^{13}$.

6 Unanimité de constat alors. Soit, il reste pourtant à comprendre les modalités de formalisation de ce discours sur l'histoire étayé par Barnave. Comment ses pages, conçues au départ pour être un mémoire défensif ${ }^{14}$, se transforment en une œuvre historique, qui fait du «moi » de l'acteur révolutionnaire le ressort épistémologique de l'événement? Pour le dire autrement, il s'agit de s'intéresser aux conditions d'écriture des textes de Barnave afin de comprendre la tension créatrice qui s'instaure entre la mise en récit de l'histoire immédiate et la pratique de la politique, en concevant celle-ci en tant que principe heuristique qui permet d'étayer une pensée de l'Histoire. 


\section{L'épreuve de la rupture}

7 Au centre des luttes révolutionnaires depuis son arrivée à Versailles, Barnave n'a pas beaucoup écrit lors de son mandat. Il est alors l'homme de la tribune, c'est là qu'il construit sa renommée et son leadership, que ce soit au sein des Jacobins ou bien à l'Assemblée nationale. N'est-t-il pas la voix du triumvirat ? N'incarne-t-il pas à lui seul le projet feuillant de l'été 1791 ? À lui revient la tâche de le défendre en prononçant les discours plus importants qui marquent les étapes de la révision constitutionnelle: le 15 juillet d'abord il se prononce sur l'inviolabilité du roi, le 11 août il intervient à propos de la révision du seuil électoral, le 15 août enfin il prend la parole concernant la responsabilité ministérielle ${ }^{15}$. À chaque occasion quelques notes gribouillées suffisent à lui inspirer des idées qui sont longuement étayées en improvisant ${ }^{16}$.

8 C'est seulement après le retour dans sa ville natale, début janvier 1792, que Barnave peut prendre du recul et développer une réflexion sur les événements qui sont en train de se dérouler. Les échanges épistolaires nourris qu'il entretient avec ses anciens collègues et avec les nouveaux législateurs feuillants, sont décisifs en ce sens, en l'obligeant à la fois à reconsidérer tout son parcours depuis 1789 et à justifier les dissensions de plus en plus profondes qui le séparent de ses interlocuteurs. À Alexandre Lameth et à Adrien Duport, trop pressés de reprendre le contrôle du jeu politique qui leur échappe, il conseille d'accepter le fait que «la révolution que l'Assemblée constituante s'était flattée d'avoir consommée, n'a été qu'un songe », en rappelant par ailleurs qu'« on attendrait en vain pour fixer la révolution, un effort des gens de bien ${ }^{17}$. " Les seconds sont en revanche invités à reconnaître que, sans les jacobins auxquels ils s'opposent farouchement, l'Assemblée législative "ne serait qu'un vaisseau sans voiles » et que par conséquent il serait impossible de gouverner le Pays, sans leur énergie et leur patriotisme ${ }^{18}$.

9 Si les interlocuteurs varient, ces textes sous-entendent la même vision du phénomène révolutionnaire. Alors que quelques mois auparavant, dans sa correspondance secrète entretenue avec la reine, Barnave se montrait convaincu du fait que l'acteur historique pouvait maitriser le processus révolutionnaire, et qu'il pourrait même en infléchir la dynamique par sa propre action ${ }^{19}$, début 1792, c'est une autre vision qui prime, nettement plus pessimiste: l'individu ne peut qu'accepter la force des choses et négocier en permanence avec les circonstances.

10 Éloigné du «tourbillon révolutionnaire », comme lui-même le définit dans ses lettres, l'ancien député saisit l'occasion pour se faire observateur et pédagogue. Observateur par ses analyses détaillées des événements, pédagogue par sa volonté d'en expliquer constamment le sens caché à ses correspondants. Cette double posture l'amène progressivement à se détacher de la quotidienneté pour esquisser une réflexion sur ce que lui parait désormais l'illusion des hommes de 1789 , celle de pouvoir être les protagonistes à leur propre destin. La mise à distance du combat politique lui permet de mieux interroger le caractère de la rupture, dont il ne cesse pas de souligner la violence et l'ampleur. Pour cet ancien jacobin devenu «jeune professeur de révolution et de royauté constitutionnelle ${ }^{20} "$, la première est un déchirement au niveau politique collectif bien évidemment, mais elle l'est également dans l'ordre du biographique et dans l'ordre temporel : trois niveaux d'expérience indissolublement liés qu'il s'agit pour Barnave de déconstruire et d'ordonner à travers l'adoption d'une méthode critique capable de 
transformer cette interrogation initiale en ressort de la mise en récit de l'histoire de la Révolution.

11 Certes, de premier abord, on a du mal à saisir la place du subjectif même dans les lettres de 1792 : les réflexions politiques se succèdent à longueur de pages sans qu'il n'apparaisse une seule ligne sur leur auteur. S'il est parfois question de souligner «l'utilité de la retraite» et les «douceurs de la liberté» qui y sont associées, ces allusions s'accompagnent dialectiquement, mais inévitablement, à l'évocation de la "peine» suscitée par le fait de ne pas pouvoir «se concerter avec ses amis", de ne pas prendre part à la lutte dans la capitale. La sphère privée est racontée, sinon appréhendée par l'auteur lui-même, en tant que privation de l'action publique, comme si l'individu ne pouvait se donner à voir qu'au prisme de son activité politique. Il faut attendre une lettre à une femme, Madame Lameth, pour qu'enfin Barnave ose confier ses émotions, son désarroi même, face à la tournure prise par les événements :

Il est bien intéressant de commencer une révolution, mais il est bien à charge d'être obligé de la finir. J'ai toujours méprisé ces philanthropes qui sacrifient le bonheur de leur patrie à des spéculations chimériques pour le bonheur de l'humanité, aujourd'hui je suis tout près de désapprouver ceux qui veulent étendre leur sollicitude et leur affection au-delà d'un petit nombre de personnes ${ }^{21}$.

Un aveu d'impuissance que celui-ci qui est d'ailleurs immédiatement renié comme s'il s'agissait d'une honteuse défaillance : «Effacez, Madame, toutes ces réflexions, un jacobin ne peut les entendre sans indignation, mais excusez-moi. [...] Pendant l'intervalle du repos, je puis bien me recueillir un moment et dire : ce n'est pas le bonheur ${ }^{22}$.»

Le passage illustre bien la tension qui lie Barnave à la Révolution, le poids prépondérant que l'engagement politique a pris sur la vie intime de l'auteur, mais dévoile aussi son incapacité de se penser en dehors de l'événement historique ${ }^{23}$. Non seulement, en février 1792, Barnave choisit de se définir encore « jacobin », en dépit de son expulsion du club six mois auparavant ${ }^{24}$, mais l'éloignement de la scène publique est vécu comme un « intervalle », avant évidemment de reprendre le combat. La Révolution demeure ainsi le seul horizon d'attente d'une vie qui avait acquis une signification inédite au moment de l'écroulement de l'Ancien Régime.

Dans les lettre de Barnave, la participation aux affaires publiques se configure en effet comme une deuxième naissance, la seule qui compte véritablement dans la mesure où elle est à la fois l'aboutissement de tout le passé et un arrachement à celui-ci. Un aboutissement car, Barnave l'avoue lui lui-même, «appelé dès ma première jeunesse à l'étude des lois par la profession de mon père, un attrait puissant dirigea toute mon attention sur le droit public. À peine connaissais-je quelques éléments des lois civiles que j'avais déjà lu et extrait la plupart des ouvrages français qui existaient alors sur les lois politiques ${ }^{25}$.» Un arrachement également, car cette vocation précoce prend un sens nouveau dès 1787 , lorsque :

La pensée de voir ma patrie affranchie de ses fers, et la caste à laquelle j'appartenais relevée de l'état d'humiliation à laquelle un gouvernement insensé semblait la condamner plus que jamais, exalta toutes les facultés de mon âme, me remplit d'ardeur et d'enthousiasme, je dévouai mon existence à la cause de la liberté et je consacrai tous mes moments aux travaux qui pouvaient me rendre capable de la servir $^{26}$.

Si l'engagement au service de la Nation est censé dévoiler pleinement le sens de son parcours individuel, il est possible de saisir la multiplicité de nuances dont le mot « jacobin » se charge sous la plume de Barnave. Il se dépouille de sa signification primaire, 
qui renvoie à l'appartenance au club de la rue Saint Honoré, pour se charger en revanche d'une connotation plus intime: il témoigne d'un attachement irréductible à une communauté idéale définie par des valeurs et par une expérience ineffaçables, par-delà des clivages politiques contingents. Toutefois, ceux-ci ne peuvent pas manquer d'impacter sa réflexion, car si, depuis 1787, pour Barnave, la Révolution est toute sa vie et toute sa vie est la Révolution, conséquence en est que, dès le 10 août 1792, penser l'une signifie s'arrêter sur l'impasse de l'autre.

16 Son Introduction à la Révolution est dès lors le couronnement, demeuré inachevé, d'une réflexion certes commencée avant le départ de son auteur de la capitale, mais, sous l'empreinte des circonstances, elle conquiert une dimension nouvelle. Le dérapage de la Législative et la chute de la monarchie constitutionnelle obligent Barnave non seulement à entreprendre une réflexion sur ce qui a occasionné la défaite du projet feuillant, mais l'amènent aussi à prendre les distances de l'événement auquel il s'était associé jusque-là, sans pour autant pouvoir le refuser en bloc. L'épreuve de la défaite conduit Barnave à inscrire son propre vécu dans un mouvement plus vaste pour y « rechercher les causes à long et à moyen terme qui pourraient inclure et peut-être expliquer le hasard de [sa] surprise singulière ${ }^{27}$. " C'est justement à partir de cette mise à distance que l'acteur révolutionnaire peut se faire auteur et devenir historien du temps présent, car, sur le plan méthodologique, elle se traduit en une tension réflexive qui rend possible l'appréhension et la narration des deux ${ }^{28}$.

17 En ce sens, Barnave se montre l'héritier d'une vieille tradition historiographique qui, tout au long de l'époque moderne, avait fait de l'écriture de l'histoire un domaine réservé aux hommes politiques évincés des affaires ou vaincus. Dès Machiavel et Guicciardini au début du XVI siècle, en passant par Enrico Davila et son Histoire des guerres civiles de France, pour arriver enfin à Clarendon et son Histoire de la Rébellion d'Angleterre, l'écriture de l'histoire avait souvent été occasionnée par une défaite qui poussait les anciens protagonistes à revenir sur le passé récent pour expliquer leur malheureuse trajectoire individuelle, en la sortant de l'événementiel ${ }^{29}$. Le vécu, loin d'être une limite épistémologique pour la compréhension de ce qui s'était passé, était censée être un atout, voire la condition nécessaire pour en dévoiler le sens profond. Elle revendiquait alors une portée éducative pour ceux qui accédaient au pouvoir, fussent-ils les princes ou les élites qui gouvernaient la citée ${ }^{30}$.

Lorsqu'il commence à rédiger son Introduction à la Révolution, Barnave est persuadé de cette dimension pédagogique. Preuve en est que dans l'un de ses cahiers de notes, dont la rédaction est contemporaine à celle des écrits sur la Révolution, il se pose la question quant à « l'utilité de l'histoire. » À lui de répondre qu'elle réside dans le fait « de faire connaître les hommes [de pouvoir]; de peindre les caractères des peuples [...] et d'apprendre ainsi l'art de perfectionner la politique. L'histoire est l'expérience de ceux qui gouvernent le monde : qu'elle se guide donc d'après ce but ; qu'elle présente l'analogie des effets et des causes : voilà son rôle ${ }^{31}$. " Pour l'ancien révolutionnaire, donc, écrire l'histoire ne signifie pas composer une œuvre savante, mais au contraire prendre en compte sa dimension civique dans la mesure où elle concourt au perfectionnement de la politique.

19 Appréhendée selon cet impératif, l'histoire ne doit pas être autre chose que « la peinture du tableau des idées, comparée à la peinture du tableau des sentiments, des passions ${ }^{32}$.» Ainsi faisant, elle " éclairera de plus en plus les secrets de la morale : ce ne sera point inutile dans les mains d'un [homme] politique habile ${ }^{33}$.» De facto, Barnave assume 
consciemment le rôle formatif du champ de l'expérience passée, la sienne, pour en faire une clé de lecture du futur, en revendiquant en même temps pour soi-même, le statut d'instituteur de la nation ${ }^{34}$.

Toutefois, les extraits démontrent également que, tout en légitimant son acte historiographique par la volonté d'en faire un instrument regni, Barnave intègre cette conception classique de l'histoire dans le sillage de l'historiographie des Lumières, puisqu'il inscrit son expérience individuelle dans une vision progressive et comparative du devenir historique. L'instance biographique se révèle alors seulement l'un des éléments sur lequel se fonde l'écriture de l'histoire de Barnave, ce qui l'éloigne radicalement de ses prédécesseurs. Le vécu n'est qu'une matière brute, qui est réélaborée, mise en récit grâce à un héritage et un langage historiographique à partir duquel se définit une nouvelle grille interprétative de la réalité, sans cesse réactualisée et modifiée par la contemporanéité.

\section{Réélire les héritages historiographiques des Lumières}

$21 \mathrm{Si}$, sans aucun doute, le futur révolutionnaire avait été confronté depuis sa jeunesse à l'idée de progrès par le biais des écrits des philosophes écossais et des Lumières françaises ${ }^{35}$, ses écrits de 1788 et 1789 sont toutefois habités essentiellement par des références aux travaux historiques de Montesquieu et de Mably, dont il reprend à la fois l'interprétation de l'histoire de France et les approches méthodologiques ${ }^{36}$.

D'une part, à l'aube de la Révolution, son discours critique de l'absolutisme se déploie par le recours à l'idée d'une ancienne constitution française, véritable dépôt de la liberté de la nation qu'il serait question de restaurer ${ }^{37}$. Certes, dans ses écrits, la condamnation de l'absolutisme demeure faible, rapportée à la violence des attaques qui lui sont portées par la même occasion par d'autres auteurs patriotes. Barnave se montre plus attentif au rôle joué par la monarchie dans le processus de formation la "nation française ${ }^{38}$ ", il n'empêche que sur l'essentiel, le futur révolutionnaire ne s'éloigne pas de la vulgate patriote, en présentant le but de la régénération du royaume comme le rétablissement d'un équilibre ancien, quoique sous de nouvelles formes.

D'autre part, la continuité avec l'historiographie du siècle des Lumières se retrouve également assurée par les méthodes d'enquête. Barnave plaide pour une histoire qui ne soit pas une narration du passé, mais une analyse problématique de celui-ci. À son avis, l'historien ne peut pas se contenter d'établir des faits, il doit dévoiler les règles qui président à l'action humaine, par-delà des hasards et des accidents du temps ${ }^{39}$. À lui de conclure que le seul qui se soit approché du but est Montesquieu. Avec le magistrat du Parlement de Bordeaux, Barnave partage notamment l'idée que les sujets de dignes de l'attention de l'historien doivent être les institutions et leurs évolutions au fil du temps, saisies selon une logique comparative ${ }^{40}$. Peu importent les vicissitudes des hommes qui exercent le pouvoir : son seul centre d'intérêt est la nation et ses rapports avec les autres puissances d'Europe. Là encore Barnave se montre l'héritier d'une tradition historiographique plus qu'il ne développe une vision originale sur le passé.

Dans ces écrits de la fin des années 1780, il est impossible de déceler ce qui constitue le propre de son œuvre en 1792-1793, c'est-à-dire l'absence de toute vision contractuelle de la politique et la conviction que la Révolution soit le fruit d'un progrès historique 
dépouillé de toute connotation morale. Ces deux aspects découlent de son épreuve directe de la politique, comme le prouve le fait qu'au moment de la rédaction de l'Introduction à la Révolution, l'ancien révolutionnaire parcourt à nouveau la même histoire de France (et d'Angleterre) lue auparavant, mais ce retournement vers le passé s'opère selon de nouveaux axes interprétatifs qui impliquent le dépassement des vieilles références, qui lui paraissent désormais obsolètes. Celles-ci ne suffisent plus à assurer les réponses en mesure d'expliquer la rupture instaurée par 1789. Celle-ci, par son caractère exceptionnel, lui impose de chercher dans le passé d'autres précédentes historiques capables de modéliser l'événement révolutionnaire. En effet, on voudrait :

Vainement se faire une juste idée de la grande révolution qui vient d'agiter la France en la considérant d'une manière isolée, en la détachant de l'histoire des empires qui nous environnent et des siècles qui nous ont précédés. [...] Il faut apercevoir la place que nous occupons dans un système plus étendu ${ }^{41}$.

S'il est difficile de trouver dans ces lignes qui commencent le récit de la Révolution française, une spécificité propre à Barnave, les réminiscences d'Adam Ferguson se confondant avec les accents de Voltaire ${ }^{42}$, les pages suivantes laissent en revanche transparaître le profond impact que l'Histoire de l'Angleterre sous la dynastie de Stuart de David Hume exerce sur l'ancien révolutionnaire en 1792 et $1793^{43}$.

Même s'il est impossible d'établir quand Barnave avait lu pour la première fois l'historien écossais, il n'y a aucun doute en revanche sur le fait qu'il revienne sur son œuvre, une fois de retour à Grenoble. Il la juge nécessaire à « consulter pour [comprendre] la révolution française $e^{44}$.» Comme beaucoup d'autres certes, Barnave s'appuie sur le texte de Hume pour établir des parallélismes hâtifs et de faible valeur épistémologique entre la guerre civile anglaise et les faits contemporains : il retrouve par exemple "de part et d'autre trois couches de patriotes. D'un côté, Strafford, les presbytériens et les indépendants, de l'autre, Mounier, les constitutionnels et les républicains ${ }^{45}$ » alors que "nos journaux et nos clubs correspondent à leurs prédicateurs, intermédiaires entre les chefs et le peuple ${ }^{46}$ .» Toutefois, quoique superficiel de par ses contenus, cet usage du texte de Hume illustre l'effort de conceptualisation opéré par Barnave qui, à l'aune de la leçon humienne, développe et nourrit une lecture inédite du processus révolutionnaire.

De Hume, en effet, l'ancien révolutionnaire grenoblois ne se limite pas à reprendre la structure formelle de la mise en récit. Dans l'Histoire d'Angleterre, il puise notamment deux éléments qui caractérisent son interprétation de l'événement français : l'attention portée au rôle des passions collectives et à l'affrontement en tant que ressorts involontaires du progrès d'un côté et l'idée que la liberté politique soit le résultat d'un processus sur le long terme, de l'autre côtét ${ }^{47}$. En d'autres termes, par le biais de la lecture de Hume, Barnave brise l'immédiateté de l'actualité, il «construit des médiations entre le temps présent et l'histoire qu'il en donne. [...] Le recul n'est pas une distance dans le temps requise comme préalable pour que l'histoire soit possible. C'est l'histoire qui crée le recul ${ }^{48}$. » Ainsi, dans un double jeu de miroir, sa propre expérience de la Révolution réactualise le passé anglais autant que celui-ci éclaire, du moins partiellement, la première, tandis que les deux s'intègrent au même temps dans une vision unique de l'histoire censée être capable de les expliquer mutuellement. 


\section{La Révolution ? Un accident de l'histoire, l'œuvre de l'homme} parenthèse, un accident de l'histoire qui ne pourra jamais changer le cours profond de l'évolution des sociétés. Si elle tient ses causes des circonstances, ses conséquences ultimes sont déjà inscrites dans «la nature des choses » et sont prévisibles, pourvu que l'on prenne en considération les règles du devenir historique. En effet, Barnave reprend l'idée, que l'histoire de l'Europe peut être lue au prisme d'une lente et incessante modification des institutions politiques qui sont la projection des rapports de propriété. À l'époque féodale, l'aristocratie détenait tout le pouvoir parce que la possession des terres acquises par droit de conquête, cependant l'essor des arts et du commerce a progressivement changé les rapports de force et a redistribué les positions de force dans la société. Pour Barnave, le constat s'impose: « une nouvelle distribution de richesse prépare une nouvelle distribution du pouvoir. De même que la possession des terres a élevé l'aristocratie, la propriété industrielle élève le pouvoir du peuple ${ }^{49}$.»

La nature des choses, dont l'action constante domine avec tant de supériorité l'influence des causes accidentelles, parvient nécessairement à produire son effet. À lui alors de remarquer que partout, en Angleterre comme sur le continent, la richesse créée par le travail et détenue essentiellement par le " peuple ", a permis à celui-ci de s'émanciper en se taillant une part de plus en plus importante dans la gestion des affaires ${ }^{50}$. Dans les petits États où le peuple a accumulé assez de richesses et a pu exercer le pouvoir directement, des républiques, de nature aristocratique, ont vu le jour. Dans les grands États en revanche, le peuple a mis sa puissance à disposition du monarque dans le but d'établir un fort contre-pouvoir à l'aristocratie. Ainsi, c'est toujours le Tiers État qui, par le biais des impôts, a fondé véritablement la puissance de la couronne, en lui donnant les moyens de bâtir une structure étatique et de rassembler des armées permanentes, deux éléments qui ont progressivement marginalisé les premiers tenants du pouvoir ${ }^{51}$.

ent, Barnave offre une lecture classique d'un Tiers État fidèle allié à la cause monarchique face aux tentations frondeuses de l'aristocratie. Pourtant, il s'appuie sur cette vulgate patriote pour en tirer des conclusions radicales, puisque, en poursuivant jusqu'au but de sa logique, Barnave affirme que la couronne ne possède d'autre légitimité pour gouverner que sa capacité d'assurer un équilibre entre les deux autres forces irréductiblement opposées. Véritable superstructure, la monarchie n'est qu'un outil de gouvernement dont la légitimité repose sur son aptitude à maintenir l'unité d'un système en continuelle évolution, en atténuant les chocs qui naissent inévitablement entre l'aristocratie et le Tiers ${ }^{52}$. Peut-elle les empêcher en cristallisant dans un ordre définitif? Peut-elle manquer à sa fonction de garant des droits de la nation ? L'histoire de la guerre civile anglaise prouve que non.

31 Jacques Ier voulut étaler un pouvoir que ses prédécesseurs avaient seulement exercé [mais] la force nationale était relevée, échauffée par les passions religieuses; les plus fortes oppositions se montrèrent. Tout s'exalta sous Charles Ier et l'enthousiasme de la liberté et de la religion se réunirent contre le prince ${ }^{53}$.

Ces deux facteurs rassemblèrent le peuple qui vainquit définitivement les aspirations absolutistes de la monarchie. Si l'expérience républicaine tourna court en se 
transformant en une dictature militaire, quelques décennies après, en 1688, la nation s'imposa définitivement, en obligeant la nouvelle dynastie à souscrire un pacte qui garantissait ses propres droits.

Barnave se montre conscient que des éléments peuvent retarder cet incessant déplacement des richesses et sa conséquente redistribution du pouvoir. En élève de Montesquieu, il pointe notamment l'influence du climat et le positionnement géographique des nations, deux éléments qui influencent le commerce et donc indirectement la montée en puissance du Tiers État. Cela explique les diverses formes institutionnelles qui existent en Europe. Pourtant, la véritable pierre d'achoppement à cette " progression douce et sensible » se trouve dans les résistances de l'aristocratie, qui exerce une hégémonie politico-culturelle, capable de dicter les mœurs et influencer les institutions.

C'est elle qui fait les lois, qui crée le préjugés et qui dirige les habitudes du peuple; elle a soin, sans doute de les combiner de manière à conserver toujours sa puissance, et si elle a autant d'habilité que de zèle à en calculer les moyens, elle pourra balancer longtemps, par l'énergie des institutions, l'influence des causes naturelles ${ }^{54}$.

Dans ces lignes est absent tout jugement moral et Barnave ne formule aucune condamnation de l'attitude de l'aristocratie en tant que telle : pour l'auteur, il existe une tendance naturelle des institutions et des classes sociales à perpétuer leur pouvoir sans qu'il n'y ait en cette attitude aucune connotation positive ou négative. Pourtant, c'est justement ce décalage entre les véritables détenteurs des richesses et les dépositaires officiels du pouvoir, qui rend possible une révolution. Lorsque le système est resté trop longtemps bloqué, il accumule une force qui porte à de "violentes commotions. »

Sous un certain point de vue, on peut considérer ces choses - la population, la richesse, les mœurs, les lumières - comme les éléments et la substance qui forment le corps social, et voir dans les lois et le gouvernement le tissu qui les contient et les enveloppe. Dans tout état de choses, il faut que l'un et l'autre soient en proportion de force et d'étendue ; si le tissu se dilate à mesure que la substance augmente de volume, les progrès du corps social pourront s'effectuer sans commotion violente, [...] mais si, au lieu d'une force élastique, il oppose une rigidité cassante, il arrivera un moment où toute proportion cessera et où il faudra que l'humeur soir consommée, brise son enveloppe et s'extravase ${ }^{55}$. La Révolution n'est pas un moment régénérateur issu d'un sentiment d'injustice ni la réaction à un régime despotique : elle est juste un moment de crise où les anciennes institutions, incapables de représenter un rapport de force inédit, qui est déjà en place, privés de légitimité s'écroulent d'un coup, laissant un vide dans lequel l'action politique et la violence qui l'accompagne, peuvent se déployer librement.

À ce propos, rien n'est plus instructif que les pages dédiées par Barnave aux événements du printemps et de l'été 1789. Leur succession, lue a posteriori comme l'aboutissement d'un siècle de philosophie des Lumières ${ }^{56}$, se transforme en revanche sous sa plume en une succession d'improvisations, où les États Généraux d'abord et l'Assemblée nationale ensuite ont toujours été en retard sur les événements. "L'ancien édifice s'écroula de toutes parts avant que l'Assemblée nationale eût posé les premières pierres du nouveau. C'est dans cette situation des hommes et des choses que la Constitution se caractérisa, tout comme l'issue par laquelle on devait sortir de cette espèce de chaos ${ }^{57}$. "

Ressentie comme une violence, la Révolution est restituée comme un bouleversement, un rapport de force subi, qui a obligé les élites patriotes à improviser sans cesse des solutions 
pour s'adapter à des circonstances qui les dépassaient. Barnave arrive même à utiliser le mot de "pulvérisation » en faisant référence à la crise de l'été 1789, terme qui traduit bien le vide et le désarroi auquel sont confrontés les révolutionnaires. D’ailleurs, en vain, on chercherait dans les pages conclusives de l'ouvrage la clarté d'exposition qui caractérisait la première partie de l'œuvre. Cela tient moins aux circonstances de rédaction, l'auteur se trouvait alors en prison, qu'à sa volonté d'explorer et de restituer les espaces d'attente de l'époque, en s'efforçant de justifier le décalage entre ce qui était envisageable alors et les perspectives qui se sont ouvertes depuis. Comment expliquer notamment son adhésion à "une monarchie que les événements semblent avoir condamnée » et sa contrariété "à une république une et indivisible ${ }^{58}$ ", maintenant en passe de s'affermir?

Répondre à cette question contraint Barnave à élargir la perspective, à faire évoluer son récit en construisant un équilibre constant entre l'histoire vécue de la Révolution, celle qui a eu effectivement lieu, et une histoire possible, vraisemblable de celle-ci. Le résultat est de rendre à la première non seulement son caractère ouvert, mais de dévoiler aussi la tension permanente entre l'action des protagonistes et une dynamique qui les emporte constamment. C'est justement ici que réside l'intérêt de cette deuxième partie du texte, plutôt que dans la justesse des jugements portés par Barnave sur ses contemporains, ensemble d'appréciations influencées par une évidente volonté de se justifier. L' Introduction à la Révolution restitue toute leur importance à l'imprévisibilité et à la force de la lame de fond révolutionnaire, en révélant la fragilité du processus d'affirmation de la légalité, toujours soumise aux aléas d'une légitimité contestée. Car, au moment même de son triomphe, «tout ce qui formait le parti révolutionnaire admit la nécessité d'une reconstruction totale; mais dès lors, on pouvait s'apercevoir, et il est devenu évident depuis, que [...] l'on différait beaucoup sur le mode et sur les moyens [de l'accomplir] ${ }^{59}$. " Née de l'écroulement de l'Ancien régime, la Révolution se mue en une succession de soubresauts, de mêlées, d'ambitions frustrées, lutte de factions d'autant plus radicale qu'elle se fonde sur des rapports de forces toujours changeants.

41 L'incapacité du nouveau régime à sortir de son état de pré-légitimité repose, en effet, sur le volontarisme dont font preuve ses créateurs. Ceux-ci, convaincus d'imprimer leur marque aux événements, sortent en réalité du sillage profond établi par «la nature des choses " et au lieu d'accélérer l'histoire, en détournerait le sens. C'est ainsi qu'ils préparent leur chute car, si leur action ne représente plus que leur propre ambition, leurs conflits demeurent néanmoins le premier ressort qui empêche à la Révolution de toucher à sa fin, pourtant souhaitée par tous.

Mais alors, comment y parvenir? Comment remonter le mécanisme étatique? Cette question, obsédante, revient à plusieurs reprises en structurant l'écriture barnavienne et la réponse se dessine au fil des pages, corollaire implicite de l'analyse qui se déploie au fil des pages. La Révolution ne pourra se terminer qu'au moment profond où ses protagonistes prendront conscience du mouvement de l'histoire auxquels ils sont invités à s'associer pour recomposer le social avec le politique, en reconstruisant un ordre juridique et symbolique dont la Constitution serait l'expression. Comme remarque justement Furet, selon Barnave: «L'homme peut être non pas l'acteur conscient [de l'histoire], mais l'observateur intelligent, à condition qu'il plie son esprit à l'expérience au lieu de prétendre la créer $^{60}$. » En d'autres termes, le je révolutionnaire peut s'exprimer dans la lutte révolutionnaire lorsqu'il surmonte les circonstances pour se faire garant du devenir historique. 

défendre devant le tribunal révolutionnaire en novembre 1793. En refusant les accusations d'avoir trahi la Révolution, il relit sa propre action au prisme de ce modèle qu'il a élaboré dans ces écrits :

Que pouvions-nous donc faire? Ce que nous avons fait : veiller à l'unité de l'empire [...]; déconcerter toutes les ambitions particulières, en mettant pour ainsi dire, la royauté en dépôt entre les mains de la nation française, jusqu'à ce que l'expérience et la calme lui eussent donné le temps et les moyens d'en disposer au gré de la volonté souveraine ${ }^{61}$.

Revenons sur les verbes utilisés par Barnave: veiller à, déconcerter, mettre entre les mains de la nation... Le révolutionnaire est à la hauteur du moment lorsqu'il s'efface, lorsqu'il n'agit pas de son propre gré, lorsqu'il est capable de répondre aux circonstances. Il est appelé à jouer le rôle de passeur ou, pour ainsi dire, d'interprète en comprenant d'une part le texte du social et d'autre part en le traduisant dans une langue politique nouvelle sans prétendre d'en inventer la grammaire.

Une révolution, dira-t-on, est donc impossible à fixer [...] et le mouvement ne s'y arrêtera que lorsque [...] la nation se reposera [...] et retombera sous son ancien despotisme ! S'il en est ainsi, c'est un profond aveuglement ou un grand crime que de l'entreprendre. Non, cette affreuse destinée n'est pas le résultat nécessaire d'une révolution ${ }^{62}$ !

\section{Conclusion : La défaite révolutionnaire comme moyen de dépasser la Révolution}

46 A travers le développement d'un discours sur l'histoire qui dissocie la signification de l'événement, de son cours effectif, Barnave opère une double rupture avec la représentation que la Révolution est en train de donner d'elle-même.

D'abord, en affirmant que 1789, mais les mêmes considérations peuvent être reprises à propos de la fondation de la République en 1792, est le produit à la fois du hasard dans la succession concrète des événements et du progrès historique dans ses conditions de possibilité, Barnave refuse de leur attribuer une quelconque portée métahistorique. Parlà, il les dépouille de leur vocation universaliste et téléologique. L'histoire n'est pas, à leurs yeux, la réalisation d'un dessein rationnel ou moral dont le phénomène révolutionnaire constituerait le moment le plus élevé. C'est ici que réside toute la différence de Barnave par rapport aux autres historiens à lui contemporaines, tels que Condorcet ou Rabaud de Saint Etienne ${ }^{63}$. Cette séparation radicale entre Révolution et principes implique une deuxième rupture épistémologique : le refus de l'idée que le cours de l'événement répond à une logique dictée par les circonstances, en expliquant son imprévisibilité.

Cette vision permet à Barnave de faire coïncider sa condition de vaincu avec l'éloignement de la Révolution par rapport à la nécessité historique qui l'avait enfantée. En considérant le volontarisme des acteurs historiques à la fois comme le moteur de la Révolution et comme un épiphénomène de l'Histoire, Barnave renverse la signification de sa propre défaite. Celle-ci devient une preuve de son altérité à la Révolution devenue une succession de péripéties. Défait dans l'immédiat, Barnave garde intact le sens de son propre engagement et l'inscrit dans un processus historique dont il a retracé l'évolution dans la première partie de son ouvrage. En se faisant historien, Barnave acquiert une 
légitimité plus profonde qui dépasse l'événement dont il est en train de traiter, il se met en surplomb sans le renier et, finalement, et il peut enfin juger une Révolution qui le condamne à mort.

Toutefois, insister sur la portée défensive du texte signifierait rester à l'écart de sa signification profonde, en ne saisissant pas sa valeur historiographique. De ce désenchantement face à l'idée que la Révolution soit intrinsèquement porteuse d'un progrès positif, du moins sur le plan politique, il en découle une reformulation du lien qui s'instaure entre l'écriture immédiate de l'histoire et l'action politique dans l'espace révolutionnaire, car le récit à chaud de l'événement devient une mise en garde contre l'illusion de faire de l'espace démocratique le théatre où les volontés des dépositaires de la souveraineté se déploient librement. En ce sens, Barnave remet en question le mythe de la Révolution au moment même où il est en train de se former alors que son histoire gagne, ou du moins voudrait le faire, une dimension performative. L'histoire devient le moyen à travers lequel les révolutionnaires peuvent se saisir de la véritable signification de leur propre action, en brisant la surenchère vers l'universel auquel les condamne leur manque de légitimité provenant du passé et de la tradition.

Contrairement à ce qu'écrira Marx quelques décennies après dans son 18 Brumaire de Louis-Bonaparte, "se remémorer de l'histoire du monde », pour Barnave, ne signifie pas «se leurrer » sur l'histoire qui est en train de s'accomplir ${ }^{64}$, mais en comprendre les enjeux par-delà l'écume et les vicissitudes dont elle est composée. C'est justement ici que l'acte historiographique immédiat déploie pleinement sa valeur civique, en transformant l'œuvre fragile et chaotique des révolutionnaires en un fait historique et déterminé, l'arrachant à l'exceptionnel. L'histoire immédiate est un acte de réappropriation, historiographique autant que politique. La Révolution retrouve son sens, non pas « en devenant un coefficient de mouvement mobilisant l'histoire au nom de chaque projet d'avenir ", mais, dans la mesure où, dépouillée de son aura mythique, elle est réduite à phénomène concret et historicisé ${ }^{65}$. En tant que telle, elle peut être jugée, critiquée même, mais, en prenant conscience d'elle-même, elle peut affirmer son ineffaçable altérité et l'impossibilité de tout retour au passé.

51 Pour peu qu'on y réfléchisse, on se convainc que, quoi qu'il arrive, nous ne pouvons pas cesser d'être libres, et que les principaux abus que nous avons détruits ne reparaîtront jamais. Combien faudrait-il essuyer de malheurs pour faire oublier de tels avantages ${ }^{66}$ !

\section{NOTES}

1. Pour un cadre exhaustif de ce retournement historiographique, voir introduction: https:// lrf.revues.org/1585.

2. Député puis pair de France sous la Monarchie de Juillet, Bérenger de la Drôme était proche de la famille de Barnave, dont il reçoit directement la tâche de réordonner les papiers du révolutionnaire. Antoine BARNAVE (éd. Alphonse-Marc BÉRENGER DE LA DRÔME), CEuvres de Barnave, Paris, Challamel, 1843, vol. 1, p. 1. 
3. Quant à la rupture historiographique et méthodologique introduite par Michelet: Christian DELACROIX, Les courants historiques en France XIX-XXe siècle, Paris, Gallimard, 2007, p. 58-68.

4. Ainsi se définira Barnave lui-même devant ses juges en octobre 1793. Gérard WALTER, Actes du Tribunal Révolutionnaire, Paris, Mercure de France, 1968, p. 303-320. Pour le portrait de Barnave : Antoine BARNAVE, op. cit., vol. 1, p. CV-CVI.

5. Tous les mémoires des anciens feuillants rendent des hommages à la figure de Barnave. Par exemple : Germaine DE STAËL, Considérations sur les principaux événements de la Révolution française, Paris, Delaunay, 1818, p. 301, 413 et 431.

6. D. R.-R. [Charles DURozoIR], «Barnave (Antoine-Pierre)», dans Louis Michaud (dir.), Biographie universelle ancienne et moderne, Paris, Thoisnier Desplaces, 1843 (rééd.), tome III, p. 111-118.

7. À titre d'exemple : «Et, toutefois, dans le passé même qui nous a légué de telles leçons, planent au-dessus des excès des partis quelques nobles figures d'hommes politiques dont la calme austérité n'a été altérée ni par l'énergie de leurs convictions, ni par la contagion de l'effervescence générale. Barnave est de ce nombre. S'il devint victime, ce fut sans avoir été persécuteur." Antoine BARNAVE, op. cit., vol. 1, p. III. Bérenger fut le premier à accéder et à publier une partie des papiers de Barnave. Sur la question et sur l'histoire des écrits de Barnave : Antoine BARNAVE (Patrice GUENIFfey éd.), De la Révolution et de la Constitution, Grenoble, Presses Universitaire de Grenoble, 1988, p. 28-35.

8. C'est sous ce titre, imposé par Bérenger de la Drôme, qu'est connu le principal texte historique de Barnave. En 1988, Patrice Gueniffey, qui en a soigné l'édition critique, en propose un autre : « De la Révolution et de la Constitution ». Pour les raisons de ce choix : ibidem.

9. Jean JAURÈs (éd. Albert SOBOUL), Histoire socialiste de la Révolution française, Paris, Ed. Sociales, 1968, vol. 1, p. 185.

10. Albert SOBOUL, Notes, dans Jean JAURÈs (Albert SOBOUL éd.), Histoire socialiste de la Révolution française, op. cit, p. 185-195; Antoine BARNAVE (Georges RUDÉ éd.), Introduction à la Révolution français, Paris, Armand Colin, 1971. Toujours dans le même sillage interprétatif voir aussi Rosanna ALBERTINI, Barnave e la rivoluzione : un sogno dell' entusiasmo ?, Pisa, Ets, 1980.

11. François FURET, «Barnave », dans François Furet, Mona Ozouf (dir.), Dictionnaire critique de la Révolution française, Paris, Flammarion, 1992, vol. Acteurs, p. 37-52 et Antoine BARNAVE (Emanuel CHILL introduit et annoté par), Power, property, and history. Barnave's Introduction to the French Revolution and other writing, New-York, Harper and Row, 1971.

12. François FURET, « Barnave », dans François Furet, Mona Ozouf (dir.), op. cit., p. 44.

13. Ibid., p. 43-44.

14. Nombre des commentateurs a sous-estimé ou nié, voir Chill, cet aspect pourtant réaffirmé à plusieurs reprises par Barnave dans sa correspondance et même dans le texte que l'on analysera dans les pages suivantes. Dans une lettre de protestation contre la séquestration de ses papiers, Barnave écrit: "Je sais que je n'en ai pas besoin pour repousser une condamnation judiciaire, mais j'en ai besoin pour imposer le silence à des calomnies si artificieusement répandues, j'en ai besoin pour exposer mon âme toute entière aux regards de mes juges et de mes concitoyens, $\mathrm{j}$ 'en ai besoin pour porter une grande cause, quand il en sera temps, au tribunal de l'opinion publique ». AN,W//12, dans Georges MICHON, Essai sur l'histoire du parti feuillant: Adrien Duport. Correspondance inédite de Barnave en 1792, Paris, Payot, 1924, p. 472.

15. Respectivement Jérôme mavidal, Emile LauRent (dir.), Archives Parlementaires, Première série, 1789-1799, Paris, P. Dupont puis CNRS, 1867-, t. XXVIII, p. 325-331, 15 juillet 1791 et t. XXIX, p. 365-368, 11 août 1791 et p. 448-458, 15 août 1791.

16. AN, W//13-14.

17. Lettre du 6 avril 1792 à Alexandre Lameth. Antoine BARNAVE (Alphonse-Marc BÉRENGER éd.), op. cit., vol. 3, p. 366-367.

18. Georges Michon, op. cit., p. 497. 
19. Alma SÖDERHJELM, Marie-Antoinette et Barnave, correspondance secrète, Paris, Armand Colin, 1934, p. 90-91.

20. Mona ozouf, Barnave pédagogue: l'éducation d'une reine, dans Mona ozouf, L'homme régénéré, Paris, Gallimard, p. 105 et plus en général, p. 91-115.

21. Lettre sans date. AN, W//13 dans Georges MichoN, op. cit., p. 399.

22. Ibidem.

23. À ce propos, on pourrait reprendre mutatis mutandis, les considérations de Merleau-Ponty qui, à propos d'une autre Révolution, utilise le terme hégélien de "conscience malheureuse " à propos du révolutionnaire qui « ne se reconnaît pas dans ce qu'il a produit et cependant il ne peut se désavouer sans contradiction.", Maurice MERLEAU-PONTY, Humanisme et terreur, Paris, Gallimard, 1947, p. 161.

24. François-Alphonse AULARD, La Société des Jacobins : recueil de documents pour l'histoire du club des Jacobins de Paris, Paris, Jouaust, Noblet, Quantin, 1889-1897, t. III, p. 149, 25 septembre 1791.

25. Antoine BARNAVE (Patrice GUENIFFEY éd.), op. cit., p. 111.

26. Ibid., p. 111-112.

27. Reinhart KOSELLECK, L'expérience de l'histoire, Paris, Gallimard, 1997, p. 241-242, plus largement voir p. 217-247.

28. La seconde partie de l'Introduction à la Révolution française, consacrée à l'œuvre de l'Assemblée constituante, révèle pleinement le processus de construction de l'écrit barnavien. D'abord Barnave présente une chronique des événements, puis il présente sa position en justifiant, si nécessaire, le décalage entre le cours de la Révolution et son point de vue. Cette démarche l'oblige enfin à s'interroger sur les conditions possibles de l'événement historique. Antoine BARNAVE (Patrice GUENIFFEY, éd.), op. cit., p. 110-160.

29. Pour des considérations intéressantes sur les rapports entre l'écriture de l'histoire, l'exercice du pouvoir et la défaite à partir du cas de Clarendon, voir : Martine Watson BROWNLEY, Clarendon and the rhetoric of historical form, Philadelphia, University of Pennsylvania press, 1985, p. 74-110 ; Brian WORMALD, Clarendon, politics, history and religion, 1640-1660, Cambridge, University Press, 1951, p. 228-235.

30. Ran halevi (dir.), Le savoir du Prince du Moyen Age aux Lumières. L'éducation du prince, Paris, Fayard, 2002 ; Paulina KEWES, History and Its Uses, et Blair WORDEN, Historians and poets dans Paulina Kewes (dir.), The uses of history in Early Modern England, San Marino (Calif.), Huntington library, 2006, respectivement p. 1-31 et p. 69-77.

31. Antoine BARNAVE (éd. Alphonse-Marc BÉRENGER), op. cit., vol. 4, p. 248, souligné par l'auteur de l'article.

32. Ibid., p. 249.

33. Ibidem.

34. Reinhardt KOSELLECK, Le futur passé, Paris, Ehess, 1990, p. 161-187.

35. Furet notamment insiste sur cet aspect. François FURET, «Barnave », dans François Furet, Mona Ozouf (dir), op. cit., p. 45.

36. Voir les fiches de lecture et les considérations contenues dans ses cahiers : Antoine BARNAVE (éd. Alphonse-Marc BÉRENGER), op. cit., vol. 1-3, passim. Pour l'influence de Mably : François FURET, Mona Ozouf, « Deux légitimations historiques de la société française au XVIII ${ }^{\mathrm{e}}$ siècle : Mably et Boulainvilliers ", Annales. Économies, Sociétés, Civilisations, 34e année, N. 3, 1979, p. 438-450.

37. Antoine BARNAVE, Esprit des édits : enregistrés militairement au parlement de Grenoble, le 10 mai 1788 , s.l. s.d., p. 19 et 21 ; Antoine BARNAVE, Coup d'œil sur la lettre de M. de Calonne, s. 1., 1789, p. 9, 11 et 15. Sur le sujet: Kenneth MARGERISON, Pamphlets and public opinion: the campaign for a Union of Orders in the Early French Revolution, West Lafayette (Ind.), Purdue university press, 1998 ; JeanMarie GOULEMOT, Le règne de l'histoire, Paris, Albin Michel, 1996; Reinhardt KOSELLECK, L'expérience de l'histoire, op. cit. 
38. Antoine BARnAVE, Esprit des édits, op. cit., p. 5-19. Antoine BARNAVE, Coup d'œil, op. cit., p. 15-16. 39. Ou selon les termes de Barnave, « il faut du génie pour arracher le grain d'or de l'alliage impur qui s'y est attaché. » Antoine BARNAVE (Alphonse-Marc BÉRENGER éd.), op. cit., vol. 4, p. 250. Justement Chill a défini l'histoire de Barnave comme un exemple d'histoire philosophique. Antoine BARNAVE (Emmanuel CHILL éd.), op. cit., p. 17. La bibliographie sur l'historiographie des Lumières est importante, je me limite ici à signaler quelques références: Hugh TREVOR-ROPER, History and the Enlightenment, New Haven, Yale University Press, 2010, p. 2-12; Reinhardt KOSELLECK, Le Règne de la Critique, Paris, Gallimard, 1979; Jean-Marie GOUlEMot, , Le règne de l'histoire, op. cit.; Giuseppe RICUPERATI (dir.), Historiographie et usages des Lumières, Berlin, Verl. A. Spitz, 2002 ; Chantal GRELl (dir.), Pratiques et concepts de l'histoire, Paris, Presses de l'Université de Paris-Sorbonne, 1990. Pour les usages politiques de l'histoire, Keith Michael BAKER, Au tribunal de l'opinion, Paris, Payot, 1993, p. 45-122.

40. Antoine BARNAVE (Alphonse -Marc BÉRENGER éd.), op. cit., vol. 4, p. 248.

41. Antoine BARNAVE (Patrice GUENIFFey, éd.), op. cit., p. 45.

42. Antoine BARNAVE (Emmanuel CHILL), op. cit., p. 17-19; François FURET, "Barnave ", art. cit., p. 44-52.

43. David hUME, Histoire de l'Angleterre sous la dynastie des Stuarts, Londres, s.e., 1760, 3 vol ; David HUME, Histoire d'Angleterre, depuis l'invasion de Jules César jusqu'à l'avènement de Henry VII, s.l., 1763-1765 ; David HUME, Histoire d'Angleterre, contenant la maison de Tudor, Paris, s. e., 1766. Le livre avait connu un immense succès en France et nombreuses rééditions jusqu'en 1788, tout particulièrement la partie consacrée à la guerre civile. Michel MALHERBE, Hume's reception in France , dans Peter Jones (dir.), The reception of David Hume in Europe, London, Thoemmes, 2005, p. 45-97.

44. Antoine BARNAVE (Alphonse -Marc BÉRENGER éd.), op. cit., vol. 4, p. 231 et plus généralement p. 231-273.

45. Ibid., vol. 2, p. 69.

46. Ibidem. Barnave retrouve par exemple « de part et d'autre trois couches de patriotes. D'un côté, Strafford, les presbytériens et les indépendants, de l'autre, Mounier, les constitutionnels et les républicains». Cette considération est le point de départ d'une longue liste qui retrace les ressemblances et les différences entre les deux moments de crise. Sur l'influence de Hume sur la Révolution, voir Laurence BONGIE, David Hume, Prophet of the Counter-revolution, Oxford, Clarendon, 1965. Même si une large partie de thèses de l'auteur est désormais dépassée, la richesse de l'ouvrage fait de celle-ci une référence incontournable sur le sujet.

47. Sur la pensée historique de David Hume : Richard D. DEES, « One of the Finest and the Most Subtile invention: Hume on the Governement ", dans Elizabeth Schimdt Radcliffe, A companion of Hume, Oxford, Blackwell, 2008, p. 388-404; Giuseppe Giarrizzo, David Hume politico e storico, Torino, Einaudi, 1962.

48. Antoine PROST, Douze leçons sur l'histoire, Paris, Seuil, 2010, p. 96 ; François HARTOG, Les régimes d'historicité, Paris, Seuil, 2011.

49. Antoine barnaVe, (Patrice GUeniffey, éd.), op. cit., p. 52

50. Barnave utilise ce terme dans l'acception de roturier, de Tiers État. Ibid., passim.

51. «La base de l'aristocratie est la propriété de la terre, la base de la monarchie la force publique, la base de la démocratie la richesse mobilière. Les révolutions de ces trois agents politiques ont été celle des gouvernements. », Ibid., p. 18.

52. Barnave avait déjà développé cette vision lors de ses discours à l'Assemblée nationale, notamment dans celui du 15 juillet 1791. Jérôme MAVIDAL, Emile LAURENT (dir.), op. cit., t. XXVIII, p. 335-336, 15 juillet 1791.

53. Antoine BARNAVE (Alphonse-Marc BÉRENGER éd.), op. cit., vol. 2, p. 233.

54. Antoine baRnaVe (Patrice GUeniffey éd.), op. cit., p. 51.

55. Ibid., p. 63. 
56. Voir les interprétations élaborées par Condorcet ou par Rabaud de Saint Etienne. JeanAntoine-Nicolas CONDORCET, Esquisse d'un tableau historique des progrès de l'esprit humain, Paris, Flammarion, 1988 [1795] ; Jean-Paul RABAUT SAINT-ÉTIENNE, Précis historique de la Révolution française , Paris, Didot l'Aîné, 1792.

57. Antoine BARNAVE (Patrice GUENIFFey éd.), op. cit., p. 115-116.

58. Ibid., p. 117.

59. Ibid., p. 116.

60. François fuRet, « Préface » dans Antoine baRnaVe (Patrice GUeniffey, éd.), op. cit., p. 24.

61. Antoine BARNAVE (Alphonse-Marc BÉRENGER éd.), op. cit., vol. 2, p. 382.

62. Antoine BARNAVE (Patrice GUENIFFey, éd.), op. cit., p. 154.

63. Ici je fais référence à l'ouvrage en général plutôt qu'à des passages spécifiques. Pour cette raison je n'ai pas indiqué des pages précises. Jean-Antoine-Nicolas CONDORCET, op. cit., p. ? passim ; Jean-Paul RABAUT SAINT-ÉTIENNE, op. cit., p. ?. passim

64. Karl MARX, Le 18 Brumaire de Louis-Bonaparte, Paris, Flammarion, 2007 (1852).

65. Reinhart KOSELLECK, op. cit, p. 76.

66. Antoine BARNAVE (Alphonse-Marc BÉRENGER), op. cit., vol. 1, p. CXX.

\section{RÉSUMÉS}

Les écrits sur la Révolution d'Antoine Barnave sont bien connus. De Jaurès à Furet plusieurs historiens ont déjà souligné la capacité de l'auteur de s'abstraire des contingences révolutionnaires pour replacer la rupture révolutionnaire à l'intérieur d'un parcours séculaire d'émancipation du Tiers Etat. Il restait à comprendre les conditions d'écriture de ces textes. Comment un acteur révolutionnaire peut se faire historien de l'événement auquel lui-même a participé ? Quels sont les ressorts et les enjeux de l'écriture historique en Révolution ? C'est à cette double question que souhaite répondre le présent article, en explorant la tension heuristique qui lie engagement politique et écriture de l'histoire. Après avoir retracé la formation culturelle de Barnave et les circonstances où il élabore ses textes historiques, il est question de voir comment l'expérience de la lutte politique, d'abord ,et de la défaite ensuite, obligent l'ancien révolutionnaire à concevoir et s'interroger sur les conditions qui rendent possible la réalisation de l'événement révolutionnaire. Par-là son œuvre, conçue comme une simple mémoire défensif, se transforme enfin en une réflexion originale et unique de la lutte révolutionnaire.

The writings on the Revolution of Antoine Barnave are well known. Several historians have emphasized the ability of the author to replace the revolutionary rupture within a secular path of emancipation of the Third Estate. It remained to understand the writing conditions of these texts. How a revolutionary actor can be a historian of the event itself was involved? What are the springs and the issues of historical writing in Revolution? It is this double question the autor wants to answer in this article, exploring the heuristic tension linking political commitment and writing of history. After tracing the cultural formation of Barnave and the circumstances in which he develops his historical texts, this article studies how the experience of the political struggle and defeat the old revolutionary design and question the conditions of possibility of realization of the revolutionary event. 
INDEX

Keywords : Barnave, historiography, immediate history, history writing, French Revolution Mots-clés : Barnave, historiographie, histoire immédiate, écriture de l'histoire, Révolution française

\section{AUTEUR}

FRANCESCO DENDENA

Università degli Studi de Milan 